\title{
3D Studies of Galaxies in Compact Groups
}

\author{
Claudia Mendes de Oliveira ${ }^{1}$, Sergio Torres-Flores ${ }^{2}$, \\ Philippe Amram ${ }^{3}$, Henri Plana ${ }^{4}$ and Benoit Epinat ${ }^{3}$ \\ ${ }^{1}$ Instituto de Astronomia, Geofísica e Ciências Atmosféricas da Universidade de São Paulo, \\ Cidade Universitária, CEP:05508-900, São Paulo, SP, Brazil \\ email: claudia.oliveira@iag.usp.br \\ ${ }^{2}$ Departamento de Física, Universidad de La Serena, \\ Av. Cisternas 1200 Norte, La Serena, Chile \\ ${ }^{3}$ Aix Marseille Université, CNRS, LAM (Laboratoire d'Astrophysique de Marseille) \\ UMR 7326, 13388, Marseille, France \\ ${ }^{4}$ Laboratório de Astrofísica Teórica e Observacional, Universidade Estadual de Santa Cruz, \\ Rodovia Ilhéus-Itabuna Km 16 45650-000 Ilhéus BA, Brazil
}

\begin{abstract}
Fabry-Perot data of compact group galaxies have been used to show that the TullyFisher relation in any photometric band, for galaxies with $\mathrm{v}_{\max }>100 \mathrm{~km} / \mathrm{s}$, is very similar to that for galaxies in other less dense environments. In the low-luminosity end, however, a few compact group galaxies fall above the relation apparently because they are too bright for their mass. Here we show that if the mass is properly computed from spectral energy distribution fitting or mass modelling, for the low-luminosity galaxies, their positions in the stellar-mass or baryonic Tully-Fisher relation are what is expected for a normal Tully-Fisher relation and the outlying positions observed in the B and $\mathrm{K}$ Tully-Fisher relation could be explained by brightening of the low-luminosity interacting galaxies due to strong star formation or AGN activity.
\end{abstract}

Keywords. galaxies: spiral, elliptical and lenticular - galaxies: kinematics and dynamics, galaxies: scaling relations

\section{Introduction}

There has been increased interest in the past years in the use of the Tully-Fisher relation $(\mathrm{TF})$ as a means of quantifying galaxy evolution as a function of redshift. However, before using the TF as such, one must understand how its slope and intercept changes with the density of the environment.

Galaxies in Hickson compact groups are often interacting galaxies, which show peculiarities in either their morphologies or kinematics. These are good laboratories where to study the effect of the dense environment on the Tully-Fisher relation. It is then of importance to find out if galaxies in the dense environments of compact groups follow well the TF relation of galaxies in less dense environments.

As shown in Mendes de Oliveira et al. (2003) and Torres-Flores et al. (2010), after a careful determination of the position angles, inclinations and kinematic centers of the galaxies, using Fabry-Perot velocity maps, and taking into account the presence of noncircular motions, most of the HCG galaxies lie on the B-band TF relation, with a few low-mass outliers. The low-mass galaxies tend to present a low rotational velocity for their observed luminosity or a high luminosity for their observed rotational velocity. If included in the fit, these low-mass galaxies affect strongly the determination of the slope and zero-point of the TF relation, biasing studies of the evolution of the TF with redshift. Studying the K-band, stellar and baryonic Tully-Fisher relation we can investigate if the mass of the galaxies is truncated due to interactions or if a burst of star formation 
could be strong enough to increase the luminosity of these objects, pushing them off the TF-relations.

\section{Results}

In order to compare the Tully-Fisher relation of compact group galaxies with that of galaxies in less dense environments we used the GHASP sample as a control sample. The kinematic properties of the GHASP sample was studied by using Fabry-Perot data and their Tully-Fisher relation was published in Torres-Flores et al. (2011). However, this sample is composed of mostly massive galaxies. Given that we are also interested in the location of the low-mass HCG galaxies on the Tully-Fisher relation defined by galaxies in less dense environments, we have complemented the GHASP sample with the gas-rich galaxies studied by McGaugh (2012). As McGaugh (2012) lists the stellar and gaseous mass of the gas-rich sample, these galaxies can be used as a comparison of the stellar and baryonic Tully-Fisher relations.

In the case of the HCG galaxies, B-band absolute magnitudes were estimated by using the apparent magnitudes listed in the Hickson catalogue, considering the different distances for each galaxy and correcting for Galactic extinction. A similar analysis was performed for the K-band absolute values, where the apparent magnitudes were taken from the 2MASS database. The stellar masses were derived from the K-band luminosities and the mass-to-light ratio obtained through the use of optical colors (see Bell et al. 2003). Rotational velocities for each galaxy were taken from Mendes de Oliveira et al. (2003) and Torres-Flores et al. (2010).

The results for the B-band, K-band and stellar Tully-Fisher relations are plotted in Figure 1. The main results of this analysis are: (1) the bulk of the galaxies in compact groups (those with $v_{\text {rot }}>100 \mathrm{~km} / \mathrm{s}$ ) follow the Tully-Fisher relation; (2) there are a few outliers at the low mass end for the B and K Tully-Fisher relations and if these are included in the fits they will affect both the slope and the intercept of the relation; (3) for the lowest mass galaxies in the compact groups, brightening in the $\mathrm{B}$ or $\mathrm{K}$ band due to interactions or AGN activity will affect the TF; (4) there are no outliers in the stellar and baryonic TF, once the masses of the low-mass galaxies are computed using spectral energy distribution fitting (SED) or mass modelling analysis.

\section{Conclusions}

3D kinematic studies of galaxies in compact groups have revealed the importance of measuring correctly the kinematic parameters, center, position angle and inclination of these interacting galaxies, as well as taking into account the contribution to its kinematics of non-circular motions when studying their TF relation. This is relevant not only for compact group galaxies but for interacting galaxies in general. 3D velocity maps are then a requirement when studying the evolution of galaxy properties in different environments and lookback times, given the increasing numbers of interacting galaxies with redshift.

Here we show that, in addition to the above, in order to correctly compute the slope and intercept of the TF relation for interacting galaxies one must be aware that specially for galaxies with maximum rotational velocities smaller than $100 \mathrm{~km} \mathrm{~s}^{-1}$, masses have to be obtained from SED fitting or mass modelling, given that the B or $\mathrm{K}$ magnitudes of these galaxies could be brightened by star formation or nuclear activity, biasing the results for interacting galaxies. 

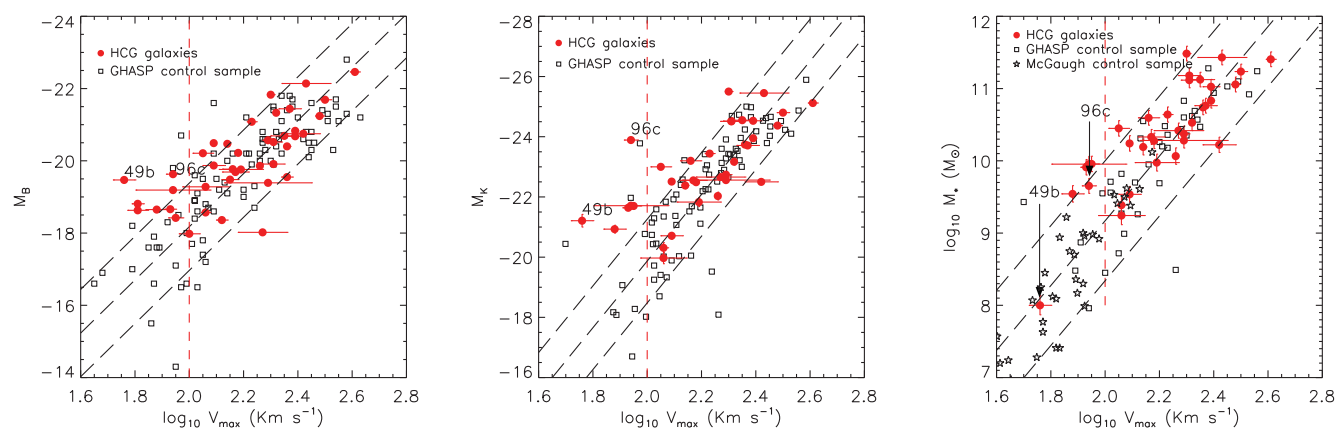

Figure 1. B-band (left-hand panel), K-band (middle-panel) and stellar (right-hand panel) TF relations for the HCG sample (red filled dots). In the three panels, we have divided the GHASP sample for galaxies that display symmetric rotation curves (filled black squares) and asymmetric rotation curves (empty squares), following Torres-Flores et al. (2011). The black dashed lines represent the linear fit and a dispersion of 1 on the GHASP data. Gas-rich galaxies (McGaugh 2012) are shown by empty stars. The red vertical lines indicate a rotational velocity of $100 \mathrm{~km}$ $\mathrm{s}^{-1}$. Note that the outliers of the relations in the first two panels follow better on the relation for the stellar TF (third pannel). The baryonic TF is shown in Torres-Flores et al. (2013).

\section{Acknowledgements}

We acknowledge support FAPESP and CNPq. ST-F also acknowledges the support of the project CONICYT PAI/ACADEMIA 7912010004

\section{References}

Bell, E. F., McIntosh, D. H., Katz, N., \& Weinberg, M. D. 2003 ApJS, 149, 289.

Mendes de Oliveira, C., Amram, P., Plana, H., \& Balkowski, C. 2003, AJ, 126, 2635.

McGaugh, S. 2012, AJ, 143, 40.

Torres-Flores S., Mendes de Oliveira, C., Amram, P., Plana, H., Epinat, B., Carignan, C., \& Balkowski, C. 2010, A\& A, 521A, 59.

Torres-Flores, S., Epinat, B., Amram, P., Plana, H., \& Mendes de Oliveira, C. 2011, MNRAS, 416, 1936.

Torres-Flores, S., Mendes de Oliveira, C., Plana, H., Amram, P., \& Epinat, B. 2013, MNRAS, 432,3085 . 